\title{
PROGRESS ON GI2T
}

\section{I.TALLON-BOSC, D.BONNEAU, A.LABEYRIE, F. MORAND, D.MOURARD, P. STEE, F. VAKILI OCA URA CNRS 1361 \\ F-O6460 Saint-Vallier de Thiey}

\begin{abstract}
The purpose of this paper is to describe the operation of GI2T, the visible Michelson stellar interferometer with two $1.5 \mathrm{~m}$ apertures, as well as a presentation of its desired evolution.
\end{abstract}

\section{Principal instrumental characteristics}

The "Grand Interféromètre à 2 télescopes" GI2T has appeared from the beginning of its construction as an original instrument with its new type of telescope. The spherical mount developed by A. Labeyrie (Labeyrie et al, 1986) has proved workable at the end of 1988 when the first stable multispeckle fringes were recorded at the common image plane. GI2T is still a prototype but continuously improved in spite of a lack of technical staff. Now in routine operation, it produces results of scientific interest, thanks to the gain of luminosity and of spectral resolution allowed by its large apertures.

Table 1 summarizes the instrumental characteristics of GI2T.

\subsection{TELESCOPES.}

The main advantages of the afocal "Boules" telescopes are their compactness and the fact that only three reflexions are necessary to bring the beams into the central station. The distance between the telescopes can be modified from two to four times for the same object during the same night, depending on the object and the scientific program. Inversely, for a given baseline and a stellar object, fringes can be recorded during three hours $(90 \mathrm{mn}$ before and after the transit time, a limitation which is due to the absence of a correction for field rotation). Therefore, an effect of partial supersynthesis can be obtained.

\subsection{BEAM RECOMBINATION.}

The telescopes being fixed on the baseline for one observation, the recombining optical system itself is moving on a carriage parallel to the baseline, in order to continuously compensate for the optical path delay between the two beams. These are recombined by superimposing the south 
and the north images after a reconfiguration of the pupils, following the classical stellar Michelson technique.

A first feature of the optical combination table is the spectral exploitation: the superimposed fringed images are dispersed, as shown in the functional diagram of Fig.1. The dispersion is of interest in three respects, if compared with a set of filters: i) the fringes are observed continuously across the spectral bandwidth; ii) the slicing of the spectra in different channels for differential contrast measurement -for ex, in the continuum and in a spectral line- can be chosen a posteriori during the data processing; iii) the change of the tilt of the fringes along the spectra with the optical path difference (OPD) provides an easy means to servo-control the position of the interference loci.

The second advantage but also difficulty of GI2T is the size of its apertures $\left(D>>r_{0}\right)$ : more light from the object is collected but it is spread over one hundred or more speckles in the image. Therefore, the optical setup encloses an image-slicer, for separating the speckles between them before their dispersion and for imaging their spectra re-arranged on the top of the detector (Bosc, 1988). The separation of the speckles is made by optically splitting the image at the entrance of the spectrograph into different slices, the width of each corresponding to one speckle. Currently, the image-slicer operates only with three slices, two for the spectrograph used for acquisition and the third for the visual tracking of the fringes.

\subsection{DETECTORS}

GI2T requires similar detectors to those developed for visible speckle interferometry, i.e. photon counting camera in order to work at very low light levels and with a short exposure time for freezing the atmospheric turbulence. The ideal detector for visible interferometry should have a good spatial resolution as well as a good temporal one. The existing available detectors result then from a compromise between the number of pixels and the minimum exposure time.

We now use at GI2T two different detectors: CP40 when it is available (an intensified 4 CCDs CCIR-standard camera) and a RANICONresistive anode detector available, which has been operating for a few months. CP40 has been built by R.Foy and A. Blazit (Blazit, 1987), RANICON by F. Paresce and his group at the Space Telescope Science Institute (Clampin et al, 1988). 


\section{Data processing}

\subsection{DATA ACQUISITION}

Both detectors provide the spatial coordinates of the centre of the photoevents; the RANICON gives also the temporal one. The centroiding numerical process, essential to compress the data, implies limitations of resolution and flux: two photon-events incoming spatially too close on CP40 or inside the dead time on the RANICON cannot be distinguished, the probability of such situations increases with the number of photo-events per temporal unit.

The data are recorded during the night on a 1,2 Gbytes hard disk before being stored on a DAT of similar capacity. Then, they can be processed off-line on the same computer, now a HP9000/710 workstation.

\subsection{PROCESSING}

The processing involves different consecutive operations (Mourard et al, 1991,1992). The preliminary operations are the correction of known and calibrated instrumental effects degrading the interferograms, as for ex. the geometrical distortion introduced by the optical set-up and the detector, and the spectral calibration using the previously recorded spectra of laboratory sources.

The instrument provides wavelength dependent contrasts. The second step of the processing consists of the choice of the spectral windows where the contrast of the fringes will be measured. This choice depends on the stellar object and the objectives of the observation.

The data consist of frames of dispersed speckles. For one exposure (fixed to $20 \mathrm{~ms}$ for CP40, adjustable for RANICON), the fringe pattern is recorded through this speckle pattern: its phase is random across the different speckles. The phase of the fringes is also randomly changing from one frame to the next. Therefore, the data processing operations must be insensitive to translation effects: the power spectrum analysis is the privileged tool. The complex contrast is directly related to the high frequency peak of the power spectrum (Roddier \& Roddier, 1976).

To calibrate each observation, the knowledge of the point spread function is necessary. We could measure it if a simultaneous observation of an unresolved star were possible. It is not easy, implying additional optics in the coudé beams and the recombining table, as proposed by A.Labeyrie (Labeyrie et al, 1987). An approximate solution consists, like in speckle interferometry, of pointing a referenced star close to the object several times during the observation . 
Some objects like the Be stars present interesting characteristics: the star itself can be assumed to be unresolved by the interferometer and its envelope is observable at certain wavelengths of Hydrogen in emission. The amplitude of the fringe modulation in the continuum, theoretically maximum, can be used as a reference to calibrate the fringes in the emission lines. Thus, the interferometer provides angular information about the circumstellar envelope, without any need of external calibration (Mourard et al, 1990).

\section{Scientific program}

To record the data sequences desired on an object takes a long time, essentially due to the moderatly good seeing conditions at the site of GI2T and the limited photon counting rate of the cameras. The data processing is delicate, due essentially to the difficulty of calibration. Therefore, an observation strategy must be defined: the object is selected if some phenomenon supposed in the models is accessible by the interferometer; the baselines, the wavelengths and nights of observation are then chosen. The ideal situation is of course to combine our observations with other "classical" techniques. Simultaneous observations by spectroscopy, photometry or speckle interferometry are necessary in order to improve the interferometric measurements on the object by complementary information.

Such operation of GI2T implies collaborations outside the instrument site, with astrophysicists building theoretical models, for the choice of the objects as well as for the interpretation of our results. One of us, P. Stee, has started to introduce the interferometric parameters into the physical models (radiative transfer in emission lines). Precious contacts have also been established in particular with M. De Groot, A.M. Hubert, H. Lamers, J. Lefevre and M. Plavec. The scientific program is based on observations of Be stars like $\gamma$ Cas, $\beta$ Lyr and oAnd, of Luminous Blue Variables like PCyg and of eclipsing binaries like $\beta$ Aur. It includes also some resolved bright stars like $\alpha$ Lyr and $\alpha$ Cyg with known angular diameter for measuring some instrumental biases.

\section{GI2T: next step?}

The data processing that we have applied up-to-now indicated some necessary improvements on the existing interferometer for an optimization of the observations and for a stronger scientific program. To accomplish that, actions must be achieved. 


\subsection{METROLOGY SYSTEM}

The positions of the telescopes are not measured with a sufficient accuracy, implying a computed position of the zero OPD shifted from the real position by a few millimetres. So, to avoid the loss of time for the research of stellar fringes, an absolute metrology prototype, using laser beam and fringe counting at several wavelengths, is being developed in the group (Morand et al, 1991) and is approaching completion. It will determine during observations the 3D-position of the centre of each telescope with an accuracy of a few microns and a response time of a few seconds. This metrology system is also developed for the OVLA project (Labeyrie et al, this conference).

\subsection{AUTOMATED FRINGE TRACKING}

The fringe detection and tracking is up-to-now visual: an observer looks continuously at several speckles dispersed by a low-dispersion spectrograph (coherence length $\approx 250 \mu \mathrm{m}$ ) and controls the motion of the optical table. The scan continues until the fringes are observed and tracked during the acquisition. Pending some improvement of the guider system, the practical magnitude is now less than only 4 or 5 , depending on the turbulence conditions and the intrinsic contrast of the fringes.

In order to increase this magnitude limit and also to be able to measure low visibility, an automated fringe tracker has been developed by L. Koechlin at Toulouse Observatory. His system processes in real-time the data recorded by the fast detector RANICON. If the application of a fast Fourier Transform algorithm on successive frames makes visible the fringe peak in a short delay $(\approx 4 s)$, a tracking of the fringes is possible, as shown by recent successful tests.

\subsection{NEW FOCAL INSTRUMENTATION.}

Concerning the optical set-up, we plan to build a new one including the necessary improvements (field rotation and atmospheric dispersion compensation, pupil stabilisation, use of the whole image) as well as a simplified unit of adaptative optics, as demonstrated by F. Roddier and his group, on each arm of the interferometer: the objective is to concentrate the light in a kernel speckle, then avoiding the present complex image slicing. A.Blazit has started the design of such a recombining optics. 


\section{Conclusion}

GI2T is now an operational prototype, that means in a period of a continuous progress. We process the data, guided by an astrophysical program; we also undertake instrumental developments. Both activities contribute to future arrays of large visible telescopes.

\section{References}

Bosc, I.: 1989, PhD Thesis, Université de Nice

Blazit, A.: 1987, Thèse d'Etat, Université de Nice

Clampin M., Crocker J., Paresce F., Rafal M.: 1988, "Optical RANICON detectors for photon counting imaging I", Review of scientific instruments 598 Part I, 1269

Labeyrie, A., Schumacher, G., Dugué, M., et al.: 1986, "Fringes obtained with the large "boules" interferometer at CERGA", Astron. Astrophys. 162, 359-364

Labeyrie A., Bosc I., Mourard D: 1987, "Multiple aperture interferometry towards the OVLA", Proceedings ESO/NOAO, Oracle, January

Morand F., Gong S, Labeyrie A. and Mourard, D.: 1991, "Status of the GI2T tri-dimensional absolute laser metrology system", Proceedings ESO Conference on High Angular Resolution Imaging, Garching, 1418 Octobre

Mourard D., Blazit A., Bonneau D., Koechlin L., Labeyrie A., Morand, F., Percheron I., Tallon-Bosc I., Vakili F.: 1991, "Status and results report on GI2T", - id-

Mourard D., Bonneau D. and Blazit A., Labeyrie A., Morand, F., Percheron I., Tallon-Bosc I., Vakili F.: 1992, "Simultaneous spectroscopic and interferometric measurements on binaries with the GI2T", Proceedings I.A.U. Coll. \#135, Atlanta, April

Mourard D., Bosc I., Labeyrie A., Koechlin L., Saha S.: 1989, "The rotating envelope of the hot star $\gamma$ Cas resolved by Optical Interferometry", Nature 342, ${ }^{\circ} 6249,520-522$

Roddier, C. and Roddier, F.: 1976, "On the fringe visibility in a Michelson stellar interferometer", J. Opt. Soc. Am. 66 (6). 
FIG. 1: Functional diagram of the recombining optical set-up

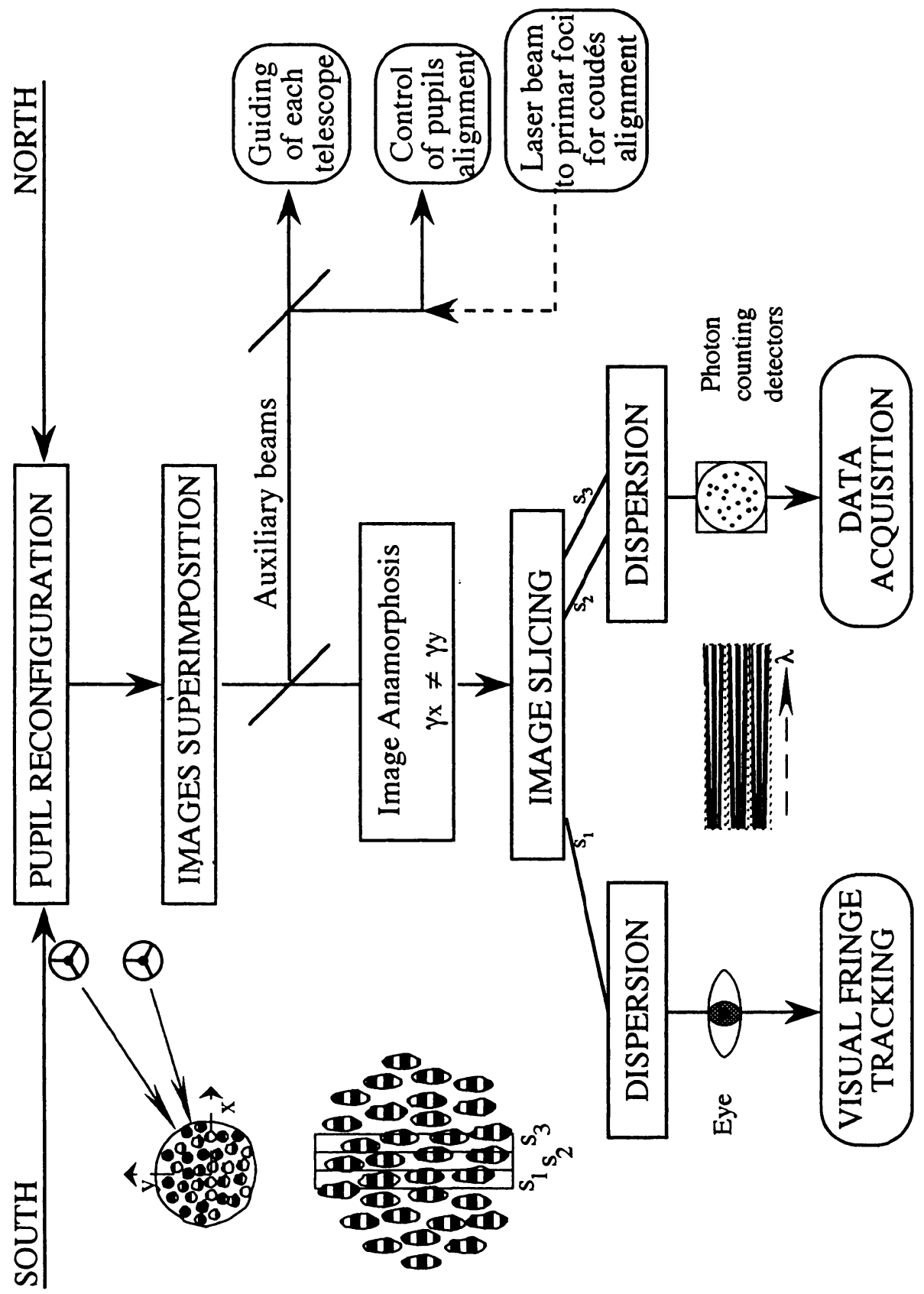

